\title{
Sensitivity of infectious SARS-CoV-2 B.1.1.7 and B.1.351 variants to neutralizing antibodies
}

\author{
Delphine Planas ${ }^{1,2,3,22}$, Timothée Bruel ${ }^{1,2,3,22}$, Ludivine Grzelak ${ }^{1,2,3,4}$, Florence Guivel-Benhassine ${ }^{1,2,3}$, \\ Isabelle Staropoli1,2,3, Françoise Porrot ${ }^{1,2,3}$, Cyril Planchais ${ }^{5}$, Julian Buchrieser (1) 1,2,3, \\ Maaran Michael Rajah ${ }^{1,2,3,4}$, Elodie Bishop ${ }^{1,2,3,4}$, Mélanie Albert ${ }^{6,7}$, Flora Donati ${ }^{6,7}$, Matthieu Prot ${ }^{8}$, \\ Sylvie Behillil ${ }^{6,7}$, Vincent Enouf ${ }^{6,7}$, Marianne Maquart ${ }^{9}$, Mounira Smati-Lafarge ${ }^{10}$, Emmanuelle Varon ${ }^{10}$, \\ Frédérique Schortgen ${ }^{11}$, Layla Yahyaoui ${ }^{12}$, Maria Gonzalez ${ }^{13}$, Jérôme De Sèze ${ }^{14,15}$, Hélène Péré16, \\ David Veyer $^{16,17}$, Aymeric Sève ${ }^{18}$, Etienne Simon-Lorière $\mathbb{1}^{8}{ }^{8}$, Samira Fafi-Kremer ${ }^{19,20}$, Karl Stefic ${ }^{9,21}$, \\ Hugo Mouquet ${ }^{5}$, Laurent Hocqueloux ${ }^{18}$, Sylvie van der Werf $\mathbb{1}^{6,7,23}$, Thierry Prazuck ${ }^{18,23}$ and \\ Olivier Schwartz $1,2,3,23 凶$
}

Severe acute respiratory syndrome coronavirus 2 (SARS-CoV-2) B.1.1.7 and B.1.351 variants were first identified in the United Kingdom and South Africa, respectively, and have since spread to many countries. These variants harboring diverse mutations in the gene encoding the spike protein raise important concerns about their immune evasion potential. Here, we isolated infectious B.1.1.7 and B.1.351 strains from acutely infected individuals. We examined sensitivity of the two variants to SARS-CoV-2 antibodies present in sera and nasal swabs from individuals infected with previously circulating strains or who were recently vaccinated, in comparison with a D614G reference virus. We utilized a new rapid neutralization assay, based on reporter cells that become positive for GFP after overnight infection. Sera from 58 convalescent individuals collected up to 9 months after symptoms, similarly neutralized B.1.1.7 and D614G. In contrast, after 9 months, convalescent sera had a mean sixfold reduction in neutralizing titers, and $40 \%$ of the samples lacked any activity against B.1.351. Sera from 19 individuals vaccinated twice with Pfizer Cominarty, longitudinally tested up to 6 weeks after vaccination, were similarly potent against B.1.1.7 but less efficacious against B.1.351, when compared to D614G. Neutralizing titers increased after the second vaccine dose, but remained 14-fold lower against B.1.351. In contrast, sera from convalescent or vaccinated individuals similarly bound the three spike proteins in a flow cytometry-based serological assay. Neutralizing antibodies were rarely detected in nasal swabs from vaccinees. Thus, faster-spreading SARS-CoV-2 variants acquired a partial resistance to neutralizing antibodies generated by natural infection or vaccination, which was most frequently detected in individuals with low antibody levels. Our results indicate that B1.351, but not B.1.1.7, may increase the risk of infection in immunized individuals.

S ARS-CoV-2 variants have rapidly emerged in humans and supplanted ancestral strains $s^{1-5}$. Their proposed increased rates of interindividual transmission conferred a replication advantage at the population level. One of the first identified variants includes the D614G mutation in the gene encoding the spike (S) protein, which enhances viral infectivity and shifts $\mathrm{S}$ protein conformation toward an angiotensin-converting enzyme 2 (ACE2)-binding fusion-competent state, without significantly modifying sensitivity to antibody neutralization ${ }^{1,6-8}$. More recently, novel variants have appeared in multiple countries, with combinations of mutations and deletions in the receptor-binding domain (RBD) and N-terminal domain of S protein, as well as in other proteins. The B.1.1.7 variant emerged in the United Kingdom, the B.1.351 variant (also termed 501Y.V2) in South Africa and the P.1 and P.2 lineages in Brazil ${ }^{2,3,5,9-12}$. Although distinct, the variants share common characteristics, including known escape mutations that were previously identified under antibody pressure selection in vitro ${ }^{2,3,13-17}$. Some of the mutations or deletions were also identified in immunocompromised individuals with prolonged infectious viral shedding and treated with convalescent plasma or S-protein

\footnotetext{
'Virus \& Immunity Unit, Department of Virology, Institut Pasteur, Paris, France. ${ }^{2}$ CNRS UMR 3569, Paris, France. ${ }^{3}$ Vaccine Research Institute, Créteil, France. ${ }^{4}$ Université de Paris, Sorbonne Paris Cité, Paris, France. ${ }^{5}$ Laboratory of Humoral Immunology, Department of Immunology, Institut Pasteur, INSERM U1222, Paris, France. 'Molecular Genetics of RNA Viruses, Department of Virology, Institut Pasteur CNRS UMR 3569, Université de Paris, Paris, France. ${ }^{7}$ National Reference Center for Respiratory Viruses, Institut Pasteur, Paris, France. ${ }^{8} \mathrm{G} 5$ Evolutionary Genomics of RNA Viruses, Institut Pasteur, Paris, France. ${ }^{9}$ INSERM U1259, Université de Tours, Tours, France. ${ }^{10} \mathrm{CHI}$ de Créteil, Service de Biologie Médicale, Créteil, France. " $\mathrm{CHI}$ de Créteil, Service de Réanimation, Créteil, France. ${ }^{12} \mathrm{CHI}$ de Créteil, Service des Urgences, Créteil, France. ${ }^{13} \mathrm{CHU}$ de Strasbourg, Service de Pathologie Professionnelle et Médecine du Travail, Strasbourg, France. ${ }^{14} \mathrm{Centre} d$ 'investigation Clinique INSERM 1434, CHU Strasbourg, France. ${ }^{15} \mathrm{CHU}$ de Strasbourg, Service de Neurologie, Strasbourg, France. ${ }^{16}$ INSERM, Functional Genomics of Solid Tumors (FunGeST), Centre de Recherche des Cordeliers, Université de Paris and Sorbonne Université, Paris, France. ${ }^{17}$ Hôpital Européen Georges Pompidou, Service de Virologie, Paris, France. ${ }^{18} \mathrm{CHR}$ d'Orléans, Service de maladies infectieuses, Orléans, France. ${ }^{19} \mathrm{CHU}$ de Strasbourg, Laboratoire de Virologie, Strasbourg, France. ${ }^{20}$ Université de Strasbourg, INSERM, IRM UMR_S 1109, Strasbourg, France. ${ }^{21} \mathrm{CHRU}$ de Tours, National Reference Center for HIV-Associated laboratory, Tours, France. ${ }^{22}$ These authors contributed equally: Delphine Planas, Timothée Bruel. ${ }^{23}$ These authors jointly supervised this work: Sylvie van der Werf, Thierry Prazuck, Olivier Schwartz.

凶e-mail: olivier.schwartz@pasteur.fr
} 
monoclonal antibodies ${ }^{3,17-19}$, indicating that antibody-escape mutations are selected in vivo. The sensitivity to antibody neutralization varies with the viral variant. B.1.1.7 seems to be more sensitive to neutralization than B.1.351. The RBD mutation N501Y, which increases affinity to ACE2 and is present in B.1.1.7 and B.1.351 $\left(\right.$ ref. ${ }^{20}$ ), does not impair on its own post-vaccine serum neutralization $^{21}$. It has been suggested that the other mutations in B.1.1.7 do not result in immune evasion of linear epitopes ${ }^{22}$. Mutations in the B.1.351 and P.1 strains, including E484K and K417N/T, are of high concern, since they partly compromise neutralization generated by previous infection or vaccination ${ }^{23-25}$ or may increase inherent viral fitness. The Pfizer Cominarty (also termed BNT162b2) vaccine-elicited human sera neutralizes SARS-CoV-2 lineage B.1.1.7 pseudovirus, with slightly reduced titers in some vaccinees, when compared to the Wuhan reference strain ${ }^{26,27}$. The Moderna mRNA-1273 vaccine also induces neutralizing antibodies to SARS-CoV-2 variants with, however, a five- to ten-fold reduction in efficacy against the B.1.351 S protein, when compared to pseudovirus bearing the D614G mutation ${ }^{27,28}$.

Neutralization efficacy has so far mostly been assessed using vesicular stomatis virus (VSV)-derived or lentivirus-derived pseudovirus assays, or with infectious SARS-CoV-2 carrying point mutations in S protein. A recent report using infectious B.1.351 virus showed that plasma samples from six convalescent donors were strongly attenuated against this strain, with half-maximal inhibitory concentration $\left(\mathrm{IC}_{50}\right)$ values 6 - to 200 -fold higher than ancestral virus ${ }^{29}$. It is thus of utmost importance to use authentic variant strains in addition to pseudovirus particles, since mutations outside of the S protein may impact inherent viral fitness and/or sensitivity to antibodies. Here, we compared the sensitivity of three authentic SARS-CoV-2 strains, the preexisting D614G virus and the B.1.1.7 and B.1.351 variants, to antibody neutralization.

\section{Results}

Isolation and characterization of SARS-CoV-2 B.1.1.7 and B.1.351 variants. We isolated the two variants from nasal swabs of individuals with infection diagnosed by quantitative PCR with reverse transcription (RT-qPCR) and sequence-diagnosed infection. The viruses were amplified by one to two passages on Vero cells. Sequences of the outgrown viruses confirmed the identity of B.1.1.7 and B.1.351 variants. Viral stocks were titrated using S-Fuse reporter cells $\mathrm{s}^{30}$. These cells are derived from a human immortalized line, termed U2OS-ACE2 ${ }^{+}$cells. They carry the GFP-split complementation system, in which two cells separately produce half of the reporter protein, producing GFP only upon fusion. Following infection, the cells produce $S$ protein at their surface and fuse with neighboring cells, generating a GFP signal as soon as $6 \mathrm{~h}$ after infection $^{30}$ (Fig. 1a). The number of $\mathrm{GFP}^{+}$cells correlated with the viral inoculum (Fig. 1b). Neutralizing SARS-CoV-2 monoclonal antibodies (mAbs) targeting the RBD can be classified into four main categories $^{31,32}$. Using the S-Fuse assay, we tested the sensitivity of the three viral strains to two RBD antibodies, mAb102 and mAb48, derived from convalescent individuals. The antibodies belong to the first category and act by blocking binding of the 'up' conformation of RBD to ACE2 (ref. ${ }^{32}$ ). MAb102 efficiently and similarly neutralized the three viral strains, with an $\mathrm{IC}_{50}$ of $\simeq 0.01 \mu \mathrm{g} \mathrm{ml}^{-1}$ (Fig. 1c,d). MAb48 neutralized D614G $\left(\mathrm{IC}_{50}\right.$ of $0.1 \mu \mathrm{g} \mathrm{ml} \mathrm{m}^{-1}$ ) but was inactive against B.1.1.7 and B.1.351 variants (Fig. 1c,d). These results confirm that the two variants can selectively display reduced sensitivity to certain antibodies. Of note, the activity of the monoclonal antibodies was similar at different viral inocula, within a range of 50 to $200 \mathrm{GFP}^{+}$syncytia per well (Fig. 1e), indicating that potential variations in the number of infected S-Fuse cells do not impact the calculation of $\mathrm{IC}_{50}$. Thus, for further studies, we selected a multiplicity of infection leading to about 150 syncytia per well for each viral strain.
Sensitivity of D614G, B.1.1.7 and B.1.351 variants to sera from convalescent individuals. We assessed the neutralization ability of sera from convalescent individuals. We selected samples from 28 donors in a longitudinal cohort of individuals with SARS-CoV-2 from the French city of Orléans (Supplementary Table 1). All individuals were diagnosed with SARS-CoV-2 infection by RT-qPCR or serology and including those who had critical, severe and mild-to-moderate coronavirus disease 2019 (COVID-19). They were not vaccinated. A total of 25 individuals were sampled twice: first, at a median of $89 \mathrm{~d}$ (range $77-105 \mathrm{~d}$ ) post onset of symptoms (POS; 3-month samples (M3)), and second, at a median of $179 \mathrm{~d}$ (range 168-197d) POS (6-month samples (M6). We incubated serially diluted sera with D614G, B.1.1.7 or B.1.351 strains, added the mixture to S-Fuse cells, and scored the $\mathrm{GFP}^{+}$cells after overnight infection. We then calculated the median effective dose $\left(\mathrm{ED}_{50}\right)$ for each combination of serum and virus. A representative example with the same donor is depicted in Fig. 2a, and the results with all donors appear in Fig. 2b. The D614G and B.1.1.7 strains were similarly sensitive to sera. At $\mathrm{M} 3$, the $\mathrm{ED}_{50}$ values were $1.5 \times 10^{3}$ and $1 \times 10^{3}$ for D614G and B.1.1.7 variants, respectively, with huge variations between individuals (from $10^{2}$ to $2 \times 10^{4}$ ), and the values did not strongly decline at M6. As expected, individuals with critical disease displayed higher neutralizing activities than those with severe or mild-to-moderate symptoms (Extended Data Fig. 1). Again, there was no significant difference between the two viral strains in each category of symptoms. For B1.351, the neutralization titers were significantly decreased by five and ten-fold at the two time points, when compared with D614G and B.1.1.7 strains (Fig. 2b and Extended Data Fig. 1).

We confirmed these results in sera from 30 individuals from another cohort of RT-qPCR-confirmed unvaccinated health-care workers from Strasbourg University Hospital who experienced mild disease ${ }^{33,34}$. The samples were collected at a later time point (M9), with a median of 233 d (range 206-258d) POS (Supplementary Table 1). Overall, the neutralization activity was lower (one representative example is shown in Fig. 2a and the results of all donors are available in Fig. 2b). There was no significant difference in neutralization between D614G and B.1.1.7 strains, with a similar $\mathrm{ED}_{50}$ of $2 \times 10^{2}$. In sharp contrast, the neutralizing activity against B.1.351 was particularly low at this late time point, with a median $\mathrm{ED}_{50}$ of 50 , representing a fourfold decrease when compared to D614G (Fig. 2b).

We then arbitrarily classified the individuals as neutralizers (with neutralizing antibodies detectable at the first serum dilution of 1:30) and non-neutralizers, for the three viral strains and the two cohorts (Fig. 2c). Most individuals neutralized the three strains at M3. The fraction of neutralizers started to decline at M6, a phenomenon which was more marked for B.1.351. The fraction of neutralizers was higher in the second cohort, with $93 \%$ of individuals neutralizing either D614G or B.1.1.7 strains at M9. In contrast, only $63 \%$ of individuals neutralized the B.1.351 strain (Fig. 2c).

Antibody binding to cells expressing D614G, B.1.1.7 and B.1.351 $S$ proteins. We next examined the binding capacity of monoclonal antibodies and sera from the convalescent individuals described above to $\mathrm{S}$ proteins from different lineages. To this aim, we adapted the flow cytometry-based S-Flow assay, which we previously established to measure the levels of antibodies binding to cells expressing the Wuhan S protein ${ }^{35}$. We transiently transfected human epithelial HEK293T (referred to as 293T) cells with plasmids expressing the D614G, B.1.1.7 and B.1.351 S proteins. Similar surface levels of the viral proteins were detected with $\mathrm{mAb} 10$, a non-neutralizing pan-coronavirus antibody targeting a conserved epitope in the S2 domain and isolated from a convalescent individual (C. Planchais et al., unpublished data; Fig. $3 \mathrm{a}$ and Supplementary Fig. 1). Moreover, the cells fused when cocultivated with ACE2-expressing cells, indicating that the three proteins were functional (data not 


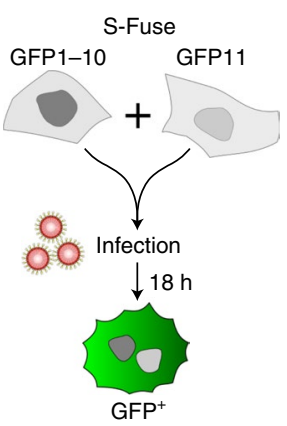

b

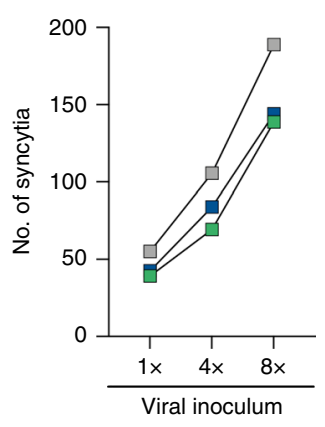

c

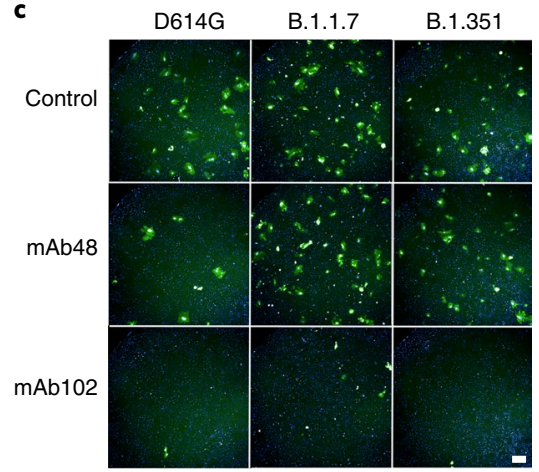

d

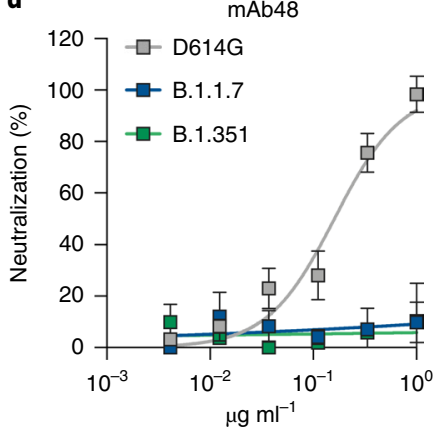

mAb102

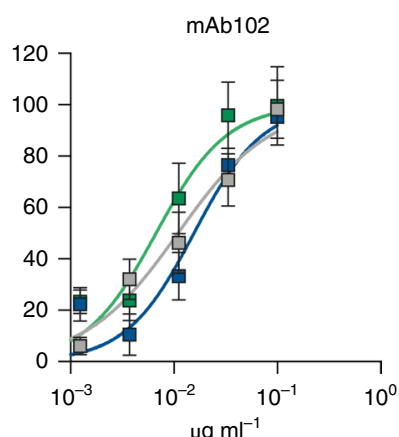

e

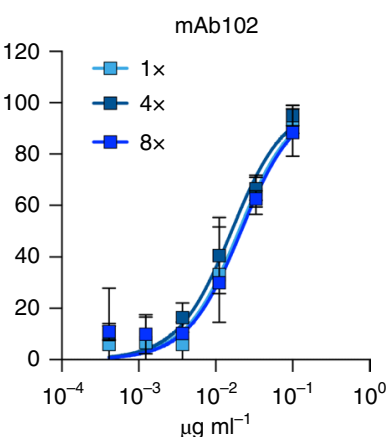

Fig. 1 | Effect of two SARS-CoV-2 monoclonal antibodies on D614G, B.1.1.7 and B1.351 strains. a, Principle of the S-Fuse reporter assay. S-Fuse cells, a mix of U20S-ACE2-GFP1-10 and U20S-ACE2-GFP11, were cocultured at a 1:1 ratio and infected with SARS-CoV-2. Cells fused upon infection and generated a GFP signal. Infection was quantified by measuring the number of GFP+ syncytia at 18h. b, Linearity of the assay with D614G (gray), B.1.1.7 (blue) and B.1.351 (green) strains. S-Fuse cells were exposed to serial dilutions of viral stocks and infection was quantified by high-content imaging. c, Representative images (from five independent experiments) of S-Fuse cells infected with D614G, B.1.1.7 and B1.351 strains in the presence or absence of two SARS-CoV-2 monoclonal antibodies (mAb48 and mAb102). Scale bar, $400 \mu \mathrm{m}$. d, Dose-response analysis of the neutralization by mAb102 and mAb48 on the three viral strains. e, Neutralization of SARS-CoV-2 (B.1.1.7) by mAb102 at three viral inocula. Results are shown as the mean \pm standard deviation (s.d.) from four independent experiments.

shown). mAb48 efficiently bound to D614G S proteins, slightly less potently to B.1.1.7, and lost all binding activity to B.1.351. Therefore, the escape of mAb48 neutralization by the two variants is due to mutations decreasing or abrogating antibody binding to its target. mAb102 bound similarly to the three S proteins, in accordance with its cross-reactive neutralizing activity (Fig. 3a,b). The N501Y mutation enhances RBD affinity to ACE2, when tested with recombinant proteins and yeast surface display ${ }^{20}$. Using flow cytometry, we assessed by the binding of a labeled soluble ACE2 protein to cells expressing the different $S$ proteins. We observed a dramatic increase in binding of soluble ACE2 to B.1.1.7 and to a lesser extent to B.1.351, which both carry the N501Y mutation, when compared to D614G (Fig. 3a,b).

We next tested our panel of sera from convalescent individuals against the different $\mathrm{S}$ proteins. Overall, the sera bound similarly to all three S proteins, even though B.1.351 displayed a slight reduction (statistically significant at M6) in the mean fluorescence intensity (MFI) of binding (Fig. 3c). We observed a global and slight decrease in MFI at M9, compared to samples from M3 and M6 (Fig. 3c). Altogether, these results indicate that $\mathrm{S}$ proteins from B.1.351, and to a higher extent B.1.1.7, display increased affinity for ACE2 while escaping binding to some monoclonal antibodies and either to a lesser degree or not at all to polyclonal sera.

Sensitivity of D614G, B.1.1.7 and B.1.351 variants to sera from vaccine recipients. We next asked whether vaccine-elicited antibodies inhibit infection by the different variants. In France, vaccination started in January 2020 with the Pfizer Cominarty vaccine.
We thus selected 19 vaccine recipients from a cohort of vaccinated health-care workers established in Orléans. The characteristics of vaccinees are depicted in Supplementary Table 2. Sera and nasal swabs were sampled at week 2 (17 individuals), week 3 (18 individuals), week 4 (11 individuals; corresponding to week 1 after the second dose), week 5 (16 individuals; corresponding to week 2 after the second dose) and week 6 (15 individuals; corresponding to week 3 after the second dose), with a median of 13, 19, 28, 34 and $41 \mathrm{~d}$ after the first dose, respectively. This allowed us to assess the early humoral response to vaccination. We first analyzed 16 of 19 vaccinees that were not previously infected with SARS-CoV-2, as assessed by the absence of preexisting S-protein and anti-nucleoprotein $(\mathrm{N})$ antibodies. A representative example of the evolution of the neutralizing response in one vaccine recipient at the four time points is depicted in Fig. 4a. In this instance, the serum only neutralized D614G at week 2, whereas B.1.1.7 started to be neutralized at week 3, although less efficiently than D614G. B.1.1.7 and D614G strains were similarly neutralized at week 4 . The anti-B.1.351 response was negative up to week 3 , and became detectable at week 4 , at a level lower than the two other viruses.

The $\mathrm{ED}_{50}$ results from sera of the 16 vaccine recipients at the four time points are presented in Fig. $4 \mathrm{~b}$, whereas the evolution of their neutralization titers over the 6 weeks after vaccination appears in Fig. 4c. We also arbitrarily classified individuals as neutralizers (with neutralizing antibodies detectable at a serum dilution of 1:30) and non-neutralizers, for the three viral strains (Fig. 4d). Two weeks after vaccination, antibodies neutralizing the D614G strain were detected in sera from 5 of 15 recipients (33\%; with an 
a

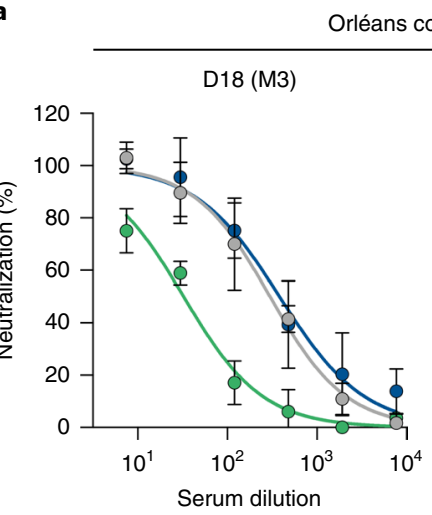

rléans cohort (M3-M6)
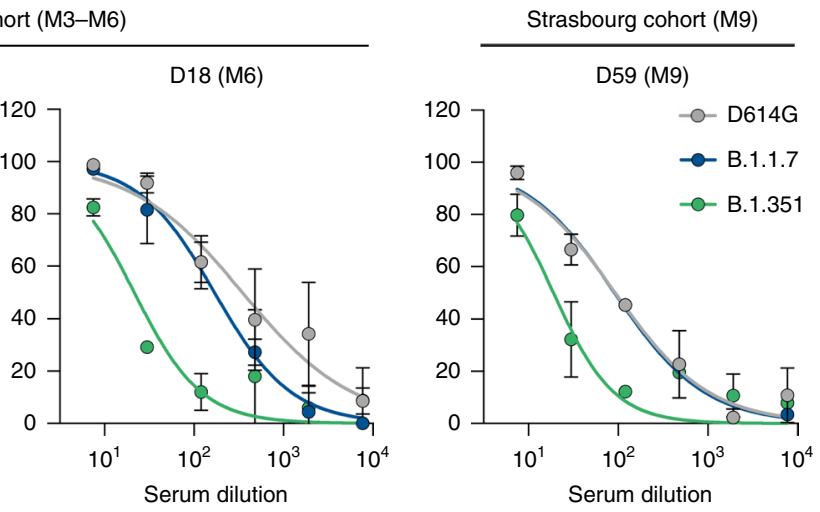

b
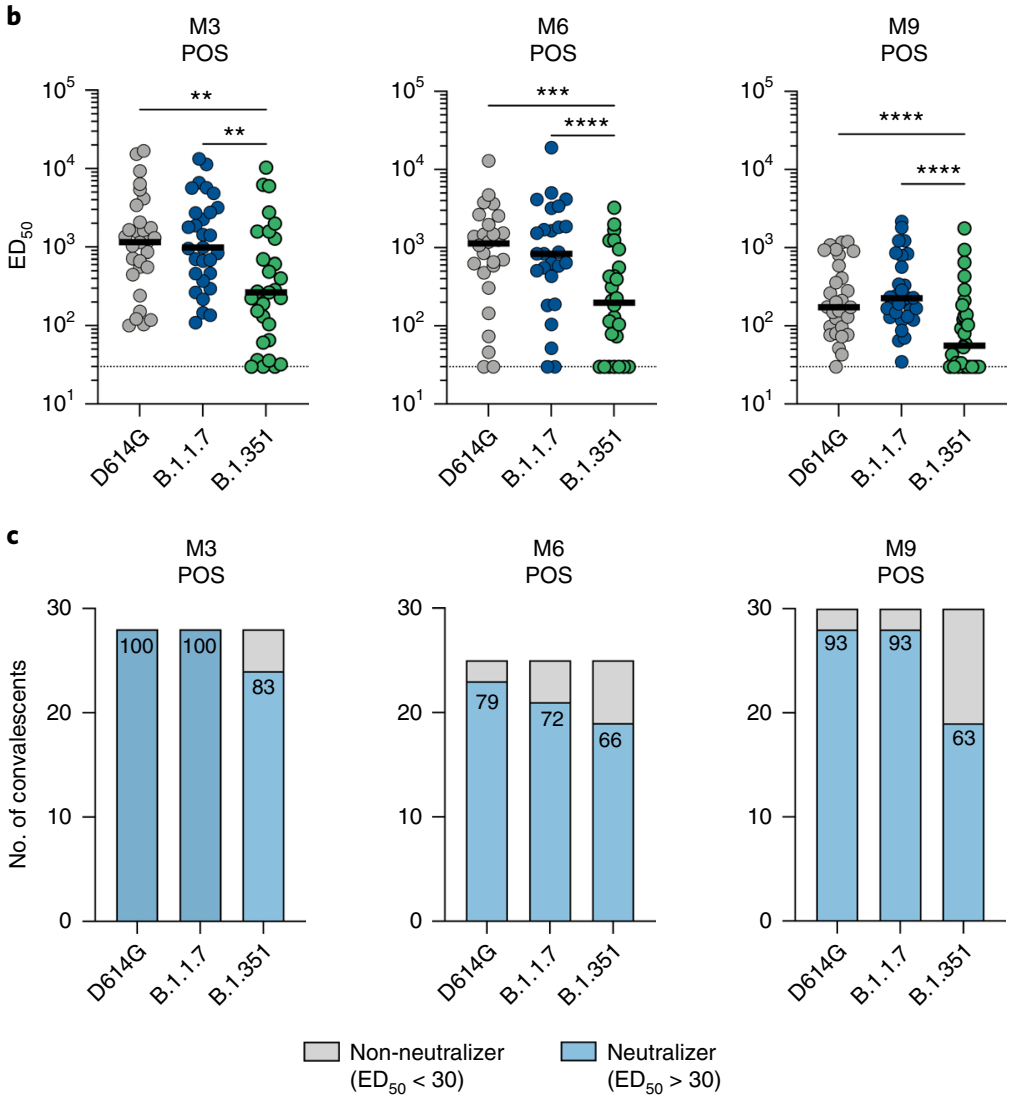

Fig. 2 | Sensitivity of SARS-CoV-2 D614G, B.1.1.7 and B.1.351 variants to sera from convalescent individuals. a, Examples of neutralization curves with sera from two donors (D18 and D59). The first donor, from the Orléans cohort, was sequentially sampled at months 3 (M3) and 6 (M6; left and middle, respectively). The second donor, from the Strasbourg cohort, was sampled at month 9 (M9; right). Results are shown as the mean \pm s.d. from three independent experiments. $\mathbf{b}, \mathrm{ED}_{50}$ of neutralization of the three viral isolates. Sera from the Orléans cohort were sequentially sampled at M3 ( $n=28$ ) and M6 ( $n=25$; left and middle, respectively), and 30 sera from the Strasbourg cohort were sampled at M9 (right). Data are the mean from two to four independent experiments. The dotted line indicates the limit of detection $\left(E D_{50}=30\right)$. Two-sided Friedman test with Dunn's test for multiple comparisons was performed between each viral strain at the different time points; ${ }^{\star \star} P<0.01,{ }^{\star \star \star}{ }^{*} P<0.001,{ }^{\star \star \star \star} P<0.0001$. M3: D614G versus B.1.351, $P=0.0012 ; B .1 .1 .7$ versus B.1.351, $P=0.0012$. M6: D614G versus B.1.351, $P=0.0005$; B.1.1.7 versus B.1.351, $P<0.0001$. M9: D614G versus B.1.351, $P<0.0001 ; B .1 .1 .7$ versus B.1.351, $P<0.0001$. C, Each individual was arbitrarily defined as a 'neutralizer' (blue) if neutralizing activity was detected at the first serum dilution (1:30) or 'non-neutralizer' (gray) if no activity was detected. The numbers indicate the percentage of neutralizers.

arbitrary threshold of $\mathrm{ED}_{50}>30$ for neutralization positivity; Fig. $4 \mathrm{~b}, \mathrm{~d})$. The titers were relatively modest at this early time point $\left(\mathrm{ED}_{50}\right.$ of 30). These low titers were less efficient against B.1.1.7, with 2 of 15 neutralizers (13\%), and were inactive against B.1.351 (Fig. 4b,c). At week 3, the neutralizing activity increased against D614G and B.1.1.7. There was, however, a threefold reduction in the neutralization titers against B.1.1.7 (Fig. 4b), whereas B.1.351 remained insensitive. At the last time point (week 3 after the second dose), titers increased in most of the recipients and were similar between D614G and B.1.1.7. Titers remained 14-fold and 53-fold lower against B.1.351, when compared to D614G and B.1.117, respectively (Fig. 4b,c). At week 3, 63\%, 38\% and $0 \%$ of the samples neutralized D614G, B.1.1.7 and B.1.351 strains, respectively (Fig. 4d). At week $6,92 \%$ of the samples neutralized D614G and B.1.1.7, whereas $77 \%$ neutralized B.1.351 with a low titer (Fig. 4d). The S-Flow assay demonstrated the presence of antibodies binding to the three $S$ proteins 
a

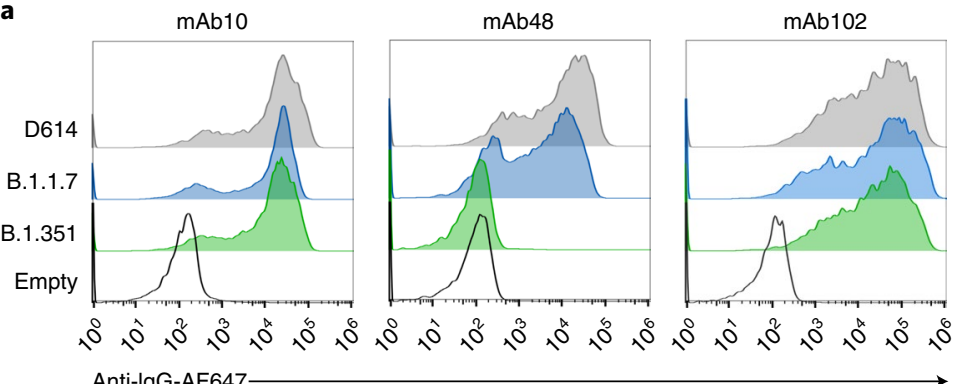

Anti-lgG-AF647

b
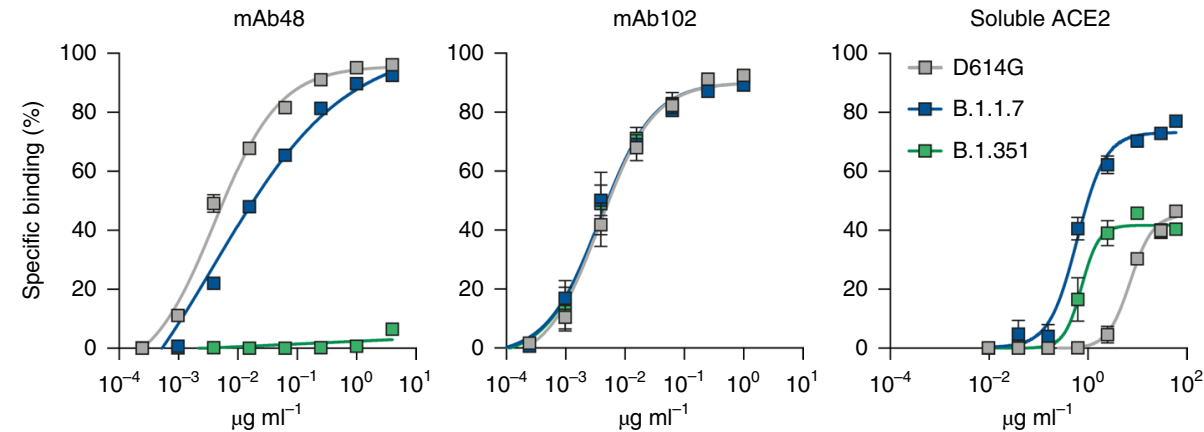

c

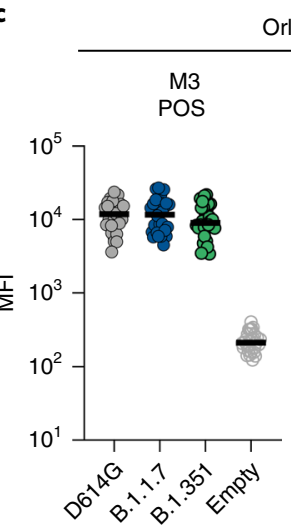

Orléans cohort
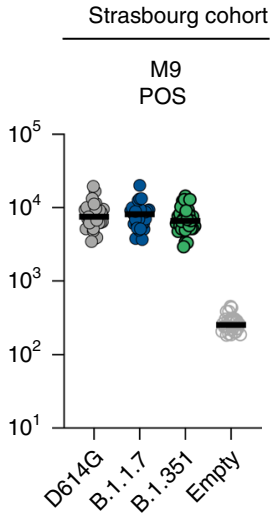

Fig. 3 | Antibody binding to cells expressing D614G, B.1.1.7 and B.1.351 S proteins. a, Binding of monoclonal antibodies or soluble ACE2. HEK293T cells were transiently transfected with plasmids expressing the D614G, B.1.1.7 and B.1.351 S proteins. After $24 \mathrm{~h}$, cells were stained with SARS-CoV-2 antibody mAb10 (a pan-coronavirus antibody), mAb48, mAb102 or soluble ACE2 (ACE2-biotin at $10 \mu g$ ml ${ }^{-1}$ revealed with fluorescent streptavidin) and analyzed by flow cytometry. One representative example of binding is shown. $\mathbf{b}$, Titration binding curves of mAb48, mAb102 and ACE2 to the three $\mathrm{S}$ proteins. Data are the mean \pm s.d. of three independent experiments. c, Binding of the panel of 83 sera from 58 convalescent individuals. Sera were tested at a 1:300 dilution. Data are the mean of two independent experiments. Two-sided Friedman test with Dunn's test for multiple comparisons was performed between each viral strain at the different time points, ${ }^{\star} P<0.05$. M6: B.1.1.7 versus B.1.351, $P=0.037$.

at the different sampling times (Extended Data Fig. 2). Therefore, the Pfizer Cominarty vaccine generated a neutralizing response that efficiently targeted D614G and B.1.1.7 with a delay in the appearance of neutralizing antibodies to B.1.1.7 and B.1.351 relative to D614G. The titers remained lower against B.1.351, even in responders. The vaccine displays a protective efficacy against COVID-19 as soon as 2 weeks after the first dose ${ }^{36}$. Our results suggest that the low neutralizing titers $\left(\mathrm{ED}_{50}\right.$ of $\left.50-100\right)$ in the sera may correspond to the observed protection against severe disease.

Sensitivity of SARS-CoV-2 B.1.1.7 and B.1.351 variants to nasal swabs from vaccine recipients. Little is known about the levels and function of vaccine-elicited antibodies in mucosal samples. We thus measured the neutralizing activity and the levels of antibodies in nasal swabs from the series of vaccine recipients (Extended Data Fig. 2). We did not detect any antiviral effect in these samples at all time points (up to 6 weeks after vaccination; Extended Data Fig. 2a,b). We also analyzed three additional vaccine recipients who were seropositive for anti-N at the time of vaccination, indicative of a previous infection. Two recipients were diagnosed with SARS-CoV-2 by PCR in March and April 2020 and experienced a mild disease. The third recipient did not report any previous signs reminiscent of COVID-19. In these three individuals, the serum neutralizing titers were strikingly high at week 2 after the first dose $\left(\mathrm{ED}_{50}\right.$ of about $10^{4}$; Extended Data Fig. $\left.2 \mathrm{c}\right)$ and remained similarly high at week 5 (data not shown). Two of the three vaccine recipients who were previously seropositive for SARS-CoV-2 displayed a low neutralizing activity in their nasal swabs, when measured at week 2 after the first dose (Extended Data Fig. 2d). Their nasal swabs neutralized D614G and B.1.117 strains similarly but were inactive against B.1.351. As a control, we analyzed ten pre-pandemic nasopharyngeal swab specimens (collected by the National Reference 
a

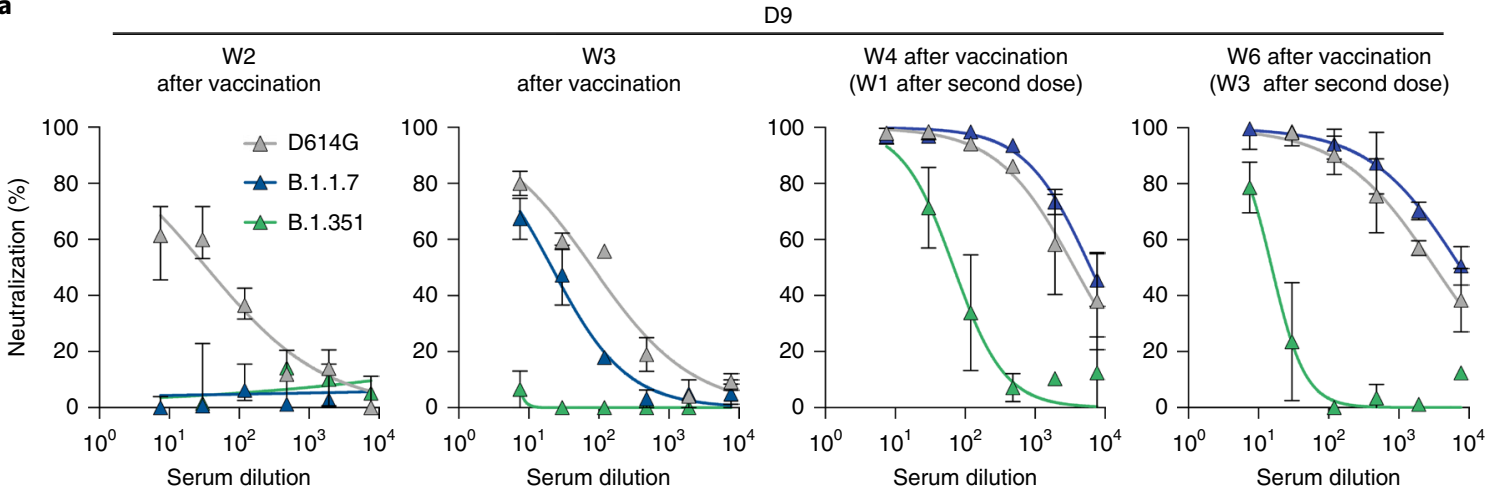

b $\quad \begin{aligned} & \text { W2 } \\ & \text { after vaccination }\end{aligned}$

vaccination

W4 after vaccination W6 after vaccination
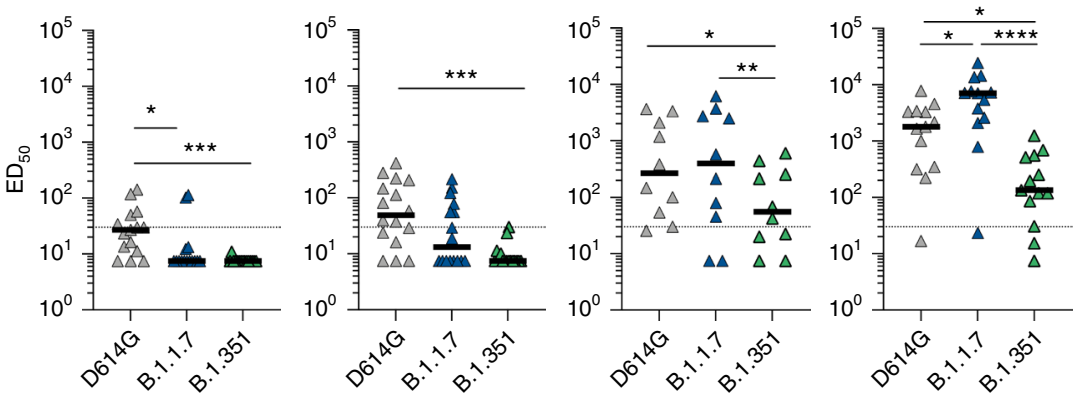

c
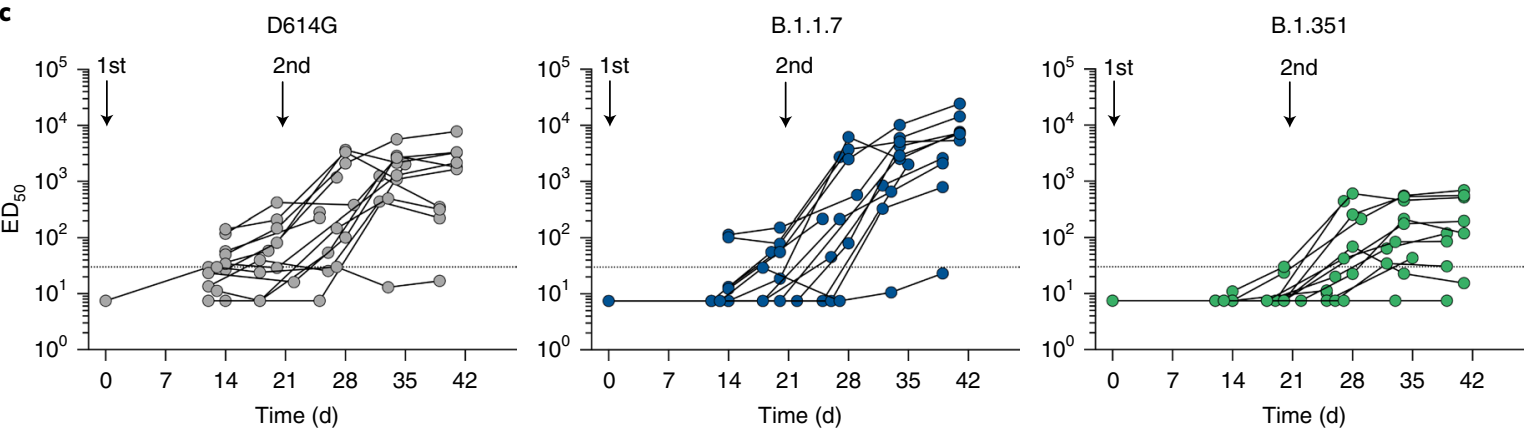

d $\quad$ W2

W3

W4 after vaccination (W1 after second dose)

W6 after vaccination

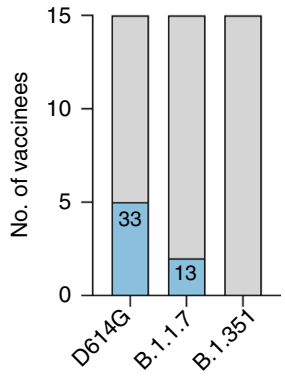

after vaccination

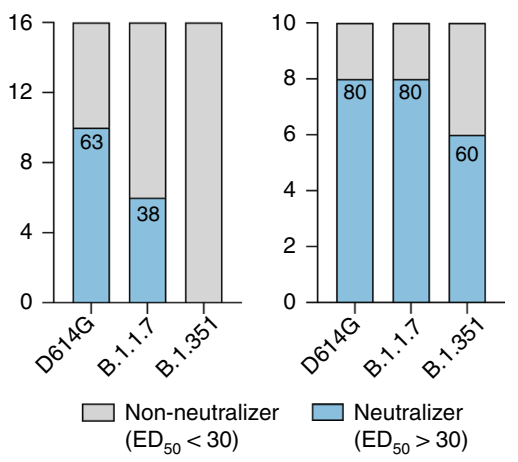

(W3 after second dose)

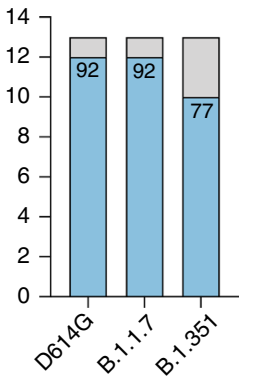

Fig. 4 | Sensitivity of SARS-CoV-2 D614G, B.1.1.7 and B.1.351 variants to sera from vaccine recipients. a, Examples of neutralization curves with sera from one donor (D9) at week 2 (W2), W3, W4 (W1 after second dose) and W6 (W3 after second dose) after vaccination. Results are shown as the mean \pm s.d. from three independent experiments. $\mathbf{b}$, Neutralization $\mathrm{ED}_{50}$ values of the three viral isolates. Sera from 10 to 16 vaccine recipients were sampled at $\mathrm{W} 2$ $(n=15), \mathrm{W} 3(n=16)$ and W1 and W3 after the second dose $(n=10$ and 15, respectively). Data are the mean from two to four independent experiments. The dotted line indicates an $\mathrm{ED}_{50}$ of 30. Two-sided Friedman test with Dunn's test for multiple comparisons was performed between each viral strain at the different time points; ${ }^{\star} P<0.05,{ }^{\star \star} P<0.01,{ }^{\star \star \star} P<0.001,{ }^{\star \star \star \star} P<0.0001$. W2: D614G versus B.1.1.7, $P=0.0105$; D614G versus B.1.351, $P=0.008$. W3: D614G versus B.1.351, $P=0.0002$. W4: D614G versus B.1.351, $P=0.011$; B.1.1.7 versus B.1.351, $P=0.0052$. W6: D614G versus B.1.1.7, $P=0.0324 ; D 614 G$ versus B.1.351, $P=0.0324 ; B .1 .1 .7$ versus B.1.351, $P<0.0001$. $\mathbf{c}$, Evolution overtime of neutralizing antibody titers in 14 vaccine recipients. The arrows indicate the first and second doses of vaccines. d, Each individual was arbitrarily defined as a 'neutralizer' (blue) if neutralizing activity was detected at a 1:30 serum dilution or 'non-neutralizer' (gray) if no activity was detected. The numbers indicate the percentage of neutralizers. 
Center in 2018 for the purpose of influenza surveillance) for the presence of neutralizing SARS-CoV-2 antibodies. The samples were all negative starting at a 1:4 dilution, ruling out a confounding nonspecific effect of a substance that may be present in the swabs (Supplementary Fig. 2). The levels of S-protein antibodies and their neutralization activity in the sera and nasal swabs of vaccine recipients are summarized in the heat map in Extended Data Fig. 2f. In the swabs, we detected SARS-CoV-2 S-protein-binding antibodies in 7 of 13 available samples at week 3, and 14 of 15 samples at week 4 (Extended Data Fig. 2f). The three previously seropositive vaccine recipients had the highest binding antibody levels in the nasal swab. Unfortunately, we did not have access to their nasal swabs before vaccination, precluding determination of whether previous infection, vaccination or, more likely, the combination of both, generated a neutralizing response in these individuals. Of note, the sera of the two previously infected and vaccinated individuals also contained neutralizing antibodies (Extended Data Fig. 2f) Altogether, our results suggest that vaccinees probably do not elicit an early humoral response detectable at mucosal surfaces. They strengthen the hypothesis that some vaccines may not protect against viral acquisition and infection of the oral-nasal region, but may prevent severe disease associated with viral dissemination in the lower respiratory tract. Our results are in line with those obtained in nonhuman primates, in which vaccinated and then challenged animals display detectable viral loads in nasal swabs but not in lower airways ${ }^{37}$.

\section{Discussion}

Here, we analyzed the cross-reactivity of the humoral response to preexisting SARS-CoV-2 viruses and recently emerging variants in sera from long-term convalescent individuals and recent vaccine recipients. We report that immune sera had slightly reduced but largely preserved activity against B.1.1.7 when compared to the reference D614G strain. The B.1.351 variant is potentially more problematic as it is less sensitive or even insensitive to sera from immunized individuals. The E484K mutation, which can mediate antibody escape of B.1.351 and P.1 variants, has been recently detected in the B.1.1.7 lineage in the United Kingdom, and thus represents a major potential threat for previously immune populations. Here, we used authentic clinical viral isolates, rather than pseudovirus, providing a relevant way to assess inherent viral fitness and the potential impact of additional mutations outside of $S$ protein on sensitivity to neutralizing antibodies. On a technical note, the combination of S-Fuse and S-Flow assays, two methods of analysis of viral infectivity, neutralization or antibody levels, allowed a rapid assessment of the properties of emerging variants. The workflow established here can be easily adapted to any novel viral strain. Potential limitations of our study are the relatively low number of individuals analyzed and the short time frame of analysis after vaccination. Furthermore, we have not investigated the impact of preexisting cellular immunity, which may be more cross-reactive against variants than the humoral response. Future work with more vaccine recipients that have or haven't been previously infected, and longer surveillance periods, will help to characterize the role of local and systemic humoral responses in vaccine efficacy. Our study also highlights the importance of the second dose of the Pfizer Cominarty vaccine, which was associated with a strong increase of neutralizing antibody titers and a widening of strain cross-reactive antibody responses. In conclusion, our results demonstrate that suboptimal or declining antibody responses are associated with a loss of cross-reactivity against novel emerging viral strains.

\section{Online content}

Any methods, additional references, Nature Research reporting summaries, source data, extended data, supplementary information, acknowledgements, peer review information; details of author contributions and competing interests; and statements of data and code availability are available at https://doi.org/10.1038/ s41591-021-01318-5.

Received: 12 February 2021; Accepted: 11 March 2021; Published online: 26 March 2021

\section{References}

1. Yurkovetskiy, L. et al. Structural and functional analysis of the D614G SARS-CoV-2 spike protein variant. Cell 183, 739-751 (2020).

2. Kemp, S. et al. Recurrent emergence and transmission of a SARS-CoV-2 spike deletion $\Delta$ H69/V70. Preprint at BioRxiv https://doi.org/10.1101/2020.12.14. 422555 (2020).

3. Kemp, S. A. et al. SARS-CoV-2 evolution during treatment of chronic infection. Nature https://doi.org/10.1038/s41586-021-03291-y (2021).

4. Oude Munnink, B. B. et al. Transmission of SARS-CoV-2 on mink farms between humans and mink and back to humans. Science 371, 172-177 (2021).

5. Tegally, H. et al. Emergence of a SARS-CoV-2 variant of concern with mutations in spike glycoprotein. Nature https://doi.org/10.1038/s41586-021 03402-9 (2021).

6. Korber, B. et al. Tracking changes in SARS-CoV-2 spike: evidence that D614G increases infectivity of the COVID-19 virus. Cell 182, 812-827 (2020).

7. Plante, J. A. et al. Spike mutation D614G alters SARS-CoV-2 fitness. Nature https://doi.org/10.1038/s41586-020-2895-3 (2020).

8. Weissman, D. et al. D614G spike mutation increases SARS-CoV-2 susceptibility to neutralization. Cell Host Microbe 29, 23-31 (2021).

9. Rambaut, A. et al. Preliminary genomic characterisation of an emergent SARS-CoV-2 lineage in the UK defined by a novel set of spike mutations. Virological https://virological.org/t/preliminary-genomic-characterisationof-an-emergent-sars-cov-2-lineage-in-the-uk-defined-by-a-novel-set-of-sp ike-mutations/563 (2020).

10. Buss, L. F. et al. Three-quarters attack rate of SARS-CoV-2 in the Brazilian Amazon during a largely unmitigated epidemic. Science 371, 288-292 (2021).

11. Faria, N. R. et al. Genomic characterisation of an emergent SARS-CoV-2 lineage in Manaus: preliminary findings. Virological https://virological.org/t/ genomic-characterisation-of-an-emergent-sars-cov-2-lineage-in-manauspreliminary-findings/586 (2021).

12. Sabino, E. C. et al. Resurgence of COVID-19 in Manaus, Brazil, despite high seroprevalence. Lancet https://doi.org/10.1016/S0140-6736(21)00183-5 (2021).

13. Weisblum, Y. et al. Escape from neutralizing antibodies by SARS-CoV-2 spike protein variants. eLife 9, e61312 (2020).

14. Andreano, E. et al. SARS-CoV-2 escape in vitro from a highly neutralizing COVID-19 convalescent plasma. Preprint at BioRxiv https://doi.org/10.1101 /2020.12.28.424451 (2020).

15. Rees-Spear, C. et al. The effect of spike mutations on SARS-CoV-2 neutralization. Cell Rep. https://doi.org/10.1016/j.celrep.2021.108890 (2021).

16. Liu, Z. et al. Identification of SARS-CoV-2 spike mutations that attenuate monoclonal and serum antibody neutralization. Cell Host Microbe 29, 477-488.e4 (2021).

17. Starr, T. N. et al. Prospective mapping of viral mutations that escape antibodies used to treat COVID-19. Science https://doi.org/10.1126/science. abf9302 (2021).

18. Avanzato, V. A. et al. Case study: prolonged infectious SARS-CoV-2 shedding from an asymptomatic immunocompromised individual with cancer. Cell https://doi.org/10.1016/j.cell.2020.10.049 (2020).

19. McCarthy, K. R. et al. Recurrent deletions in the SARS-CoV-2 spike glycoprotein drive antibody escape. Science 371, 1139-1142 (2021).

20. Zahradník, J. et al. SARS-CoV-2 RBD in vitro evolution follows contagious mutation spread, yet generates an able infection inhibitor. Preprint at BioRxiv https://doi.org/10.1101/2021.01.06.425392 (2021).

21. Xie, X. et al. Neutralization of SARS-CoV-2 spike 69/70 deletion, E484K and N501Y variants by BNT162b2 vaccine-elicited sera. Nat. Med. https://doi. org/10.1038/s41591-021-01270-4 (2021).

22. Haynes, W. A., Kamath, K., Lucas, C., Shon, J. \& Iwasaki, A. Impact of B.1.1.7 variant mutations on antibody recognition of linear SARS-CoV-2 epitopes. Preprint at medRxiv https://doi.org/10.1101/2021.01.06.20248960 (2021).

23. Wibmer, C. K. et al. SARS-CoV-2 501Y.V2 escapes neutralization by South African COVID-19 donor plasma. Nat. Med. https://doi.org/10.1038/ s41591-021-01285-x (2021).

24. Greaney, A. J. et al. Comprehensive mapping of mutations in the SARS-CoV-2 receptor-binding domain that affect recognition by polyclonal human plasma antibodies. Cell Host Microbe 29, 463-476.e6 (2021)

25. Thomson, E. C. et al. Circulating SARS-CoV-2 spike N439K variants maintain fitness while evading antibody-mediated immunity. Cell https://doi. org/10.1016/j.cell.2021.01.037 (2021). 
26. Muik, A. et al. Neutralization of SARS-CoV-2 lineage B.1.1.7 pseudovirus by BNT162b2 vaccine-elicited human sera. Science https://doi.org/10.1126/ science.abg6105 (2021)

27. Wang, P. et al. Antibody resistance of SARS-CoV-2 variants B.1.351 and B.1.1.7. Nature https://doi.org/10.1038/s41586-021-03398-2 (2021).

28. $\mathrm{Wu}, \mathrm{K}$. et al. mRNA-1273 vaccine induces neutralizing antibodies against spike mutants from global SARS-CoV-2 variants. Preprint at BioRxiv https:// doi.org/10.1101/2021.01.25.427948 (2021).

29. Cele, S. et al. Escape of SARS-CoV-2 501Y.V2 variants from neutralization by convalescent plasma. Preprint at medRxiv https://doi.org/10.1101/2021.01.26. 21250224 (2021)

30. Buchrieser, J. et al. Syncytia formation by SARS-CoV-2-infected cells. EMBO J. 39, e106267 (2020)

31. Liu, L. et al. Potent neutralizing antibodies against multiple epitopes on SARS-CoV-2 spike. Nature 584, 450-456 (2020).

32. Barnes, C. O. et al. SARS-CoV-2 neutralizing antibody structures inform therapeutic strategies. Nature 588, 682-687 (2020).
33. Fafi-Kremer, S. et al. Serologic responses to SARS-CoV-2 infection among hospital staff with mild disease in eastern France. EBioMedicine https://doi. org/10.1016/j.ebiom.2020.102915 (2020).

34. Grzelak, L. et al. Sex differences in the evolution of neutralizing antibodies to SARS-CoV-2. J. Infect. Dis. https://doi.org/10.1093/infdis/jiab127 (2021).

35. Grzelak, L. et al. A comparison of four serological assays for detecting anti-SARSCoV-2 antibodies in human serum samples from different populations. Sci. Transl. Med. https://doi.org/10.1126/scitranslmed.abc3103 (2020).

36. Polack, F. P. et al. Safety and efficacy of the BNT162b2 mRNA COVID-19 vaccine. N. Engl. J. Med. https://doi.org/10.1056/nejmoa2034577 (2020).

37. Dagotto, G., Yu, J. \& Barouch, D. H. Approaches and challenges in SARS-CoV-2 vaccine development. Cell Host Microbe 28, 364-370 (2020).

Publisher's note Springer Nature remains neutral with regard to jurisdictional claims in published maps and institutional affiliations.

(c) The Author(s), under exclusive licence to Springer Nature America, Inc. 2021 


\section{Methods}

Orléans cohort of convalescent and vaccinated individuals. Since 27 August 2020 , a prospective, monocentric, longitudinal, interventional cohort clinical study enrolling 170 SARS-CoV-2-infected individuals with different disease severities, and 30 noninfected healthy controls is ongoing, aiming to describe the persistence of specific and neutralizing antibodies over a 24 -month period. This study was approved by the Ile-de-France IV ethical committee. At enrollment, written informed consent was collected and participants completed a questionnaire covering sociodemographic characteristics, virological findings (SARS-CoV-2 RTqPCR results, including date of testing), clinical data (date of symptom onset, type of symptoms, hospitalization) and data related to anti-SARS-CoV-2 vaccination if applicable (brand product, date of first and second vaccination). Serological status of participants was assessed every 3 months. Those who underwent anti-SARS-CoV-2 vaccination had weekly blood and nasal sampling after the first dose of vaccine for a 2-month period (NCT04750720). For the present study, we selected 28 convalescent and 19 vaccinated participants. Nasal swabs were collected in the two cavities and preserved in $3 \mathrm{ml}$ of transport M4RT buffer (Remel Microtask, Thermo Fisher). Study participants did not receive any compensation. Our research complied with all relevant ethical regulations.

Strasbourg cohort of convalescent individuals. Since 17 April 2020, a prospective, interventional, monocentric, longitudinal, cohort clinical study enrolling 308 RTqPCR-diagnosed SARS-CoV-2-infected hospital staff from Strasbourg University Hospital is ongoing (NCT04441684). At enrollment, written informed consent was collected and participants completed a questionnaire covering sociodemographic characteristics, virological findings (SARS-CoV-2 RT-qPCR results including date of testing) and clinical data (date of symptom onset, type of symptoms, hospitalization). This study was approved by Institutional Review Board of Strasbourg University Hospital. The serological status of the participants has been described at M3 and M6 POS ${ }^{33,34}$. Laboratory identification of SARS-CoV-2 was performed at least $10 \mathrm{~d}$ before inclusion by RT-qPCR testing on nasopharyngeal swab specimens according to current guidelines (Institut Pasteur; WHO technical guidance). The assay targets two regions of the viral RNA-dependent RNA polymerase (RdRp) gene with a threshold of detection of ten copies per reaction. For the present study, we randomly selected 30 individuals collected at M9. Study participants did not receive any compensation. None of the participants were vaccinated at the time of blood draws.

Pre-pandemic nasal swabs. Samples were collected as part of approved ongoing influenza virus surveillance conducted by the National Reference Center for Respiratory Viruses at Institut Pasteur. The laboratory investigations were carried out in accordance with the General Data Protection Regulation (EU Regulation 2016/679 and Directive 95/46/EC) and the French data protection law (Law 78-17 on 6 January 1978 and Décret 2019-536 on 29 May 2019), which does not require a review by an ethics committee for the secondary use of samples collected for health-care purposes. In such case, the secondary use for research is authorized if the individuals have been informed of such secondary use (article L.1211-2 of the French Public Health Code).

\section{S-Fuse neutralization assay. U2OS-ACE2 GFP1-10 or GFP 11 cells, also} termed S-Fuse cells, become $\mathrm{GFP}^{+}$cells when they are productively infected with SARS-CoV-2 $\left(\mathrm{ref}^{30}\right)$. Cells tested negative for mycoplasma. Cells were mixed (at a 1:1 ratio) and plated at $8 \times 10^{3}$ cells per well in a $\mu$ Clear 96-well plate (Greiner Bio-One). The indicated SARS-CoV-2 strains were incubated with monoclonal antibodies, sera or nasal swabs at the indicated concentrations or dilutions for $15 \mathrm{~min}$ at room temperature and added to S-Fuse cells. The nasal swabs and sera were heat inactivated $30 \mathrm{~min}$ at $56^{\circ} \mathrm{C}$ before use. Then, $18 \mathrm{~h}$ later, cells were fixed with $2 \%$ paraformaldehyde, washed and stained with Hoechst (1:1,000 dilution; Invitrogen). Images were acquired with an Opera Phenix high-content confocal microscope (PerkinElmer). The GFP area and the number of nuclei were quantified using the Harmony software (PerkinElmer). The percentage of neutralization was calculated using the number of syncytia as the value with the following formula: $100 \times(1-($ value with serum - value in 'noninfected')/(value in 'no serum' - value in 'noninfected')). Neutralizing activity of each sera was expressed as the $\mathrm{ED}_{50}$. $\mathrm{ED}_{50}$ values (in $\mu \mathrm{g} \mathrm{ml}^{-1}$ for monoclonal antibodies and in dilution values for sera and nasal swabs) were calculated using a reconstructed curve with the percentage of neutralization at the different indicated concentrations. Of note, we previously reported a correlation between neutralization titers obtained with the S-Fuse reporter assay and a pseudovirus neutralization assay ${ }^{38}$.

Virus strains. The reference D614G strain (hCoV-19/France/GE1973/2020) was supplied by the National Reference Centre for Respiratory Viruses hosted by Institut Pasteur and headed by S.v.d.W. This viral strain was supplied through the European Virus Archive goes Global (EVAg) platform, a project that has received funding from the European Union's Horizon 2020 research and innovation program under grant agreement number 653316. The variant strains were isolated from nasal swabs on Vero cells and amplified by one or two passages on Vero cells. The B.1.1.7 strain originated from an individual in Tours (France) who returned from the United Kingdom. The B.1.351 strain (CNR 202100078) originated from an individual in Créteil (France). Both individuals provided informed consent for the use of their biological materials. Titration of viral stocks was performed on Vero E6 cells, with a limiting dilution technique allowing a calculation of the $50 \%$ tissue culture infectious dose, or on S-Fuse cells. Viruses were sequenced directly on nasal swabs and after one or two passages on Vero cells.

Spike-protein expression plasmids. A codon-optimized version of the SARS-Cov-2 spike gene from Wuhan reference strain (GenBank: QHD43416.1), was transferred into the phCMV backbone (GenBank: AJ318514), by replacing the VSV-G gene. The B.1.1.7 ( $\Delta 69-70, \Delta$ Y144, N501Y, A570D, D614G, P681H, T716I, S982A and D1118H) and B.1.351 (L18F, D80A, D215G, $\Delta 242-244, \mathrm{~K} 417 \mathrm{~N}$, E484K, N501Y, D614G and A701V) spike mutations were added in silico into the codon-optimized Wuhan reference strain and were ordered as synthetic genes (GeneArt, Thermo Fisher Scientific) and transferred into the phCMV backbone. The D614G S-protein was generated by introducing the corresponding mutation in the Wuhan reference strain using Q5 Site-Directed Mutagenesis Kit (NEB).

Commercial serological assays. The serum samples from the Strasbourg cohort were first tested at Hôpital de Strasbourg using two commercial assays: (1) a CE-marked LFA assay for detection of IgM and IgG against the SARS-CoV-2 RBD of the S protein developed by Biosynex (COVID-19 BSS IgG/IgM), which has a specificity of $99 \%$ and a sensitivity of $96 \%$ for samples $>22 \mathrm{~d}$ POS; and (2) a CE-marked ELISA for detection of IgG against the full-length recombinant $\mathrm{N}$ protein from Epitope Diagnostics (EDITM Novel coronavirus COVID-19 IgG), which has a specificity of $96 \%$ and a sensitivity of $81 \%$ after $28 \mathrm{~d}$ POS. The vaccine recipients were tested for anti-S antibodies at Hôpital Européen Georges Pompidou with the following assays: SARS-CoV-2 IgG (Abbott) targeting SARS-CoV- $2 \mathrm{~N}$ protein were performed on an Architect i2000SR analyzer (Abbott). Index value threshold for positivity was set to 1.4 as recommended. Beckman Coulter Access SARS-CoV-2 IgG assays targeting the RBD were performed on a UniCel DxI 800 Access Immunoassay System (Beckman Coulter). Index value threshold for positivity was set to 1 as recommended. Qualitative results and index values were used in the analysis for both assays.

S-Flow assay. The S-Flow assay was performed as described previously ${ }^{33,35}$. Briefly, 293T cells were acquired from the American Type Culture Collection (CRL-3216) and tested negative for mycoplasma. The 293T cells were transfected with the indicated S-protein expression plasmids or a control plasmid using Lipofectamine 2000 (Life technologies). One day after, transfected cells were detached using PBS-EDTA and transferred into U-bottom 96-well plates (50,000 cells per well). Cells were incubated at $4{ }^{\circ} \mathrm{C}$ for $30 \mathrm{~min}$ with sera (1:300 dilution) or nasal swabs (1:50 dilution) in PBS containing 0.5\% BSA and $2 \mathrm{mM}$ EDTA, washed with PBS and stained using anti-IgG AF647 (1:600 dilution; Thermo Fisher). Cells were washed with PBS and fixed for 10 min using $4 \%$ paraformaldehyde. Data were acquired on an Attune Nxt instrument (Life Technologies). Stainings were also performed on control (293T-empty) cells. The specificity and sensitivity of the $\mathrm{S}$-Flow serological assay were originally assessed with the Wuhan $\mathrm{S}$ protein using 253 pre-pandemic samples and 377 RT-qPCR-confirmed SARS-CoV-2 samples. The sensitivity is $99.2 \%$ with a $95 \%$ confidence interval of $97.69-99.78 \%$ and the specificity is $100 \%(98.5-100 \%)^{33,35}$. Results were analyzed with FlowJo 10.7.1 (Becton Dickinson). After testing 253 pre-pandemic samples, the positivity of a sample was defined as a specific binding above $40 \%$. The specific binding was calculated as follows: $100 \times$ (percentage binding $293 \mathrm{~T} \mathrm{~S}$ protein - percentage binding $293 \mathrm{~T}$-empty)/(100 - percentage binding 293T-empty).

Antibodies and ACE2 ectodomain. Human SARS-CoV-2 monoclonal antibodies were cloned from S-specific blood memory B cells of convalescent individuals with COVID-19 (C. Planchais et al., unpublished data). Monoclonal antibodies mAb48 and $\mathrm{mAb} 102$ recognize the $\mathrm{RBD}$, and $\mathrm{mAb} 10$ binds to a conserved region of the $\mathrm{S} 2$ domain of the S protein. Human anti-S IgG monoclonal antibodies and His-tagged recombinant ACE2 ectodomain (amino acids 19-615) cloned into pcDNA3.1 vector were produced by transient transfection of Freestyle 293-F cells and purified by affinity chromatography as previously described ${ }^{39}$. Purified ACE2 protein was biotinylated using the EZ-Link Sulfo-NHS-Biotin kit (Thermo Fisher Scientific).

Statistical analysis. Flow cytometry data were analyzed with FlowJo v10 (TriStar). Calculations were performed using Excel 365 (Microsoft). Figures were drawn using Prism 9 (GraphPad Software). Statistical analysis was conducted using GraphPad Prism 9. Statistical significance between different groups was calculated using the tests indicated in each figure legend. No statistical methods were used to predetermine sample size. The experiments were not randomized and the investigators were not blinded to allocation during experiments and outcome assessment.

Reporting Summary. Further information on research design is available in the Nature Research Reporting Summary linked to this article.

\section{Data availability}

All data supporting the findings of this study are available within the paper and are available from the corresponding author upon request. Source data are provided 
with this paper. Viral sequences are available upon request and were deposited at GISAID (https://www.gisaid.org/) under the following numbers: hCoV-19/France/ GE1973/2020 (D614G): EPI_ISL_414631; B.1.1.7: EPI_ISL_735391; B.1.1.351: EPI_ISL 964916.

\section{References}

38. Sterlin, D. et al. IgA dominates the early neutralizing antibody response to SARS-CoV-2. Sci. Transl. Med. https://doi.org/10.1126/scitranslmed.abd2223 (2020).

39. Lorin, V. \& Mouquet, H. Efficient generation of human IgA monoclonal antibodies. J. Immunol. Methods 422, 102-110 (2015)

\section{Acknowledgements}

We thank F. Rey and L. Chakrabarti (Institut Pasteur) for critical reading of the manuscript. We thank the patients who participated in this study; members of the Virus and Immunity Unit for discussions and help; N. Aulner and the UtechS Photonic BioImaging (UPBI) core facility (Institut Pasteur), a member of the France BioImaging network, for image acquisition and analysis; and M. Backovic (Institut Pasteur) for the ACE2 construct. We thank the DRCI, CIC, Médecine du travail and Pôle de Biologie teams (CHU de Strasbourg) for the management of the Strasbourg cohort and serology testing. Work in the laboratory of O.S. is funded by Institut Pasteur, Urgence COVID19 Fundraising Campaign of Institut Pasteur, ANRS, the Vaccine Research Institute (ANR-10-LABX-77), Labex IBEID (ANR-10-LABX-62-IBEID), ANR/FRM Flash COVID PROTEO-SARS-CoV-2 and IDISCOVR. Work in UPBI is funded by grant ANR-10-INSB-04-01 and Région Ile-de-France program DIM1-Health. D.P. is supported by the Vaccine Research Institute. L.G. is supported by the French Ministry of Higher Education, Research and Innovation. The laboratory of H.M. is funded by the Institut Pasteur, the Milieu Intérieur Program (ANR-10-LABX-69-01), the INSERM, REACTing and EU (RECOVER) grants. The laboratory of S.v.d.W. is funded by Institut Pasteur, CNRS, Université de Paris, Santé publique France, Labex IBEID (ANR-10-LABX62-IBEID), REACTing, EU grant RECOVER. The laboratory of S.F.-K. is funded by
Strasbourg University Hospital (SeroCoV-HUS; PRI 7782), Programme Hospitalier de Recherche Clinique (PHRC N 2017-HUS no. 6997), the Agence Nationale de la Recherche (ANR-18-CE17-0028), Laboratoire d'Excellence TRANSPLANTEX (ANR11-LABX-0070_TRANSPLANTEX) and Institut National de la Santé et de la Recherche Médicale (UMR_S 1109). Work in CHR Orléans is funded by COREVIH Centre Val de Loire. The funders of this study had no role in study design, data collection, analysis and interpretation or writing of the article.

\section{Author contributions}

Experimental strategy, design and experiments: D.P., T.B., L.G., F.G.-B., I.S., F.P., C.P., J.B., M.M.R., E.B., S.v.d.W., T.P. and O.S.; vital materials: M.A., F.D., S.B., V.E., M.M., M.G., J.D.S., H.P., D.V., A.S., E.S.L., S.F.-K., K.S., H.M., L.H., S.v.d.W and T.P.; manuscript writing: D.P., T.B. and O.S.; manuscript editing: L.D., J.D., M.M.R., H.P., D.V., S.F.-K., K.S., H.M., L.H., S.v.d.W. and T.P.

\section{Competing interests}

L.G., I.S., T.B., J.B., F.G.-B. and O.S. are coinventors on provisional patent no. US 63/020,063 entitled 'S-Flow: a FACS-based assay for serological analysis of SARS-CoV-2 infection' submitted by Institut Pasteur.

\section{Additional information}

Extended data is available for this paper at https://doi.org/10.1038/s41591-021-01318-5. Supplementary information The online version contains supplementary material available at https://doi.org/10.1038/s41591-021-01318-5.

Correspondence and requests for materials should be addressed to O.S

Peer review information Nature Medicine thanks the anonymous reviewers for their contribution to the peer review of this work. Alison Farrell was the primary editor on this article and managed its editorial process and peer review in collaboration with the rest of the editorial team.

Reprints and permissions information is available at www.nature.com/reprints. 
a

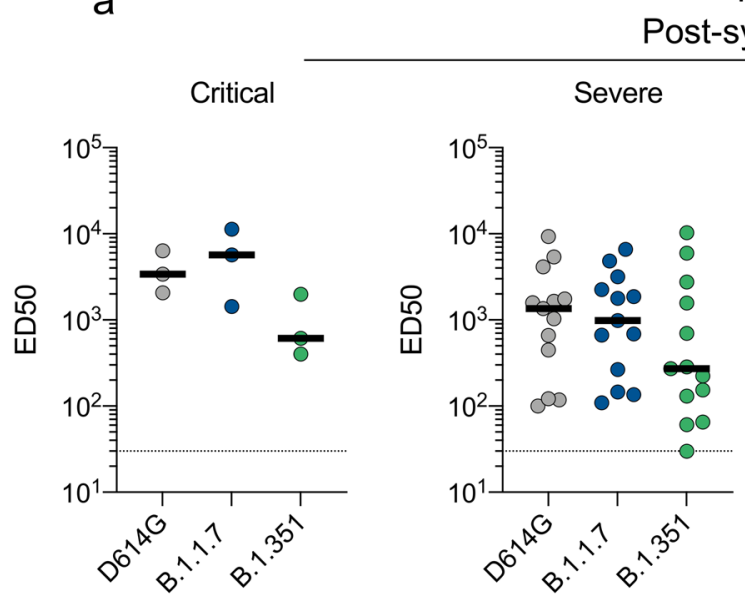

M3

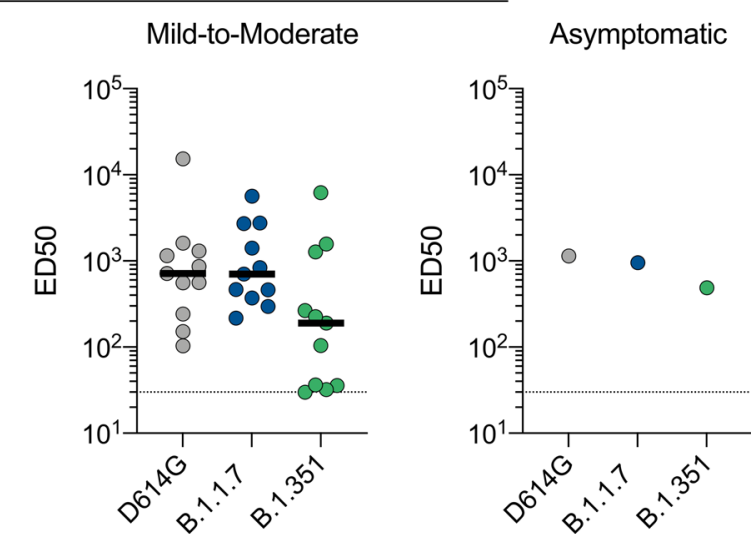

b

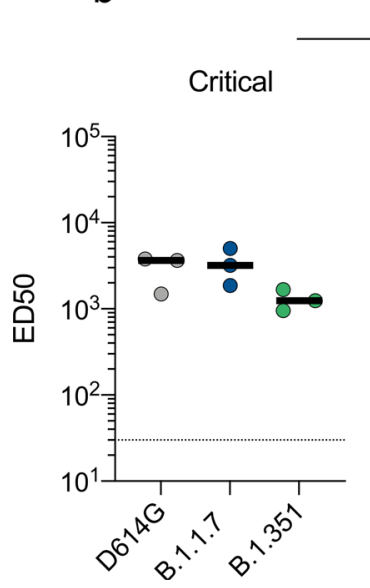

M6

Post-symptoms
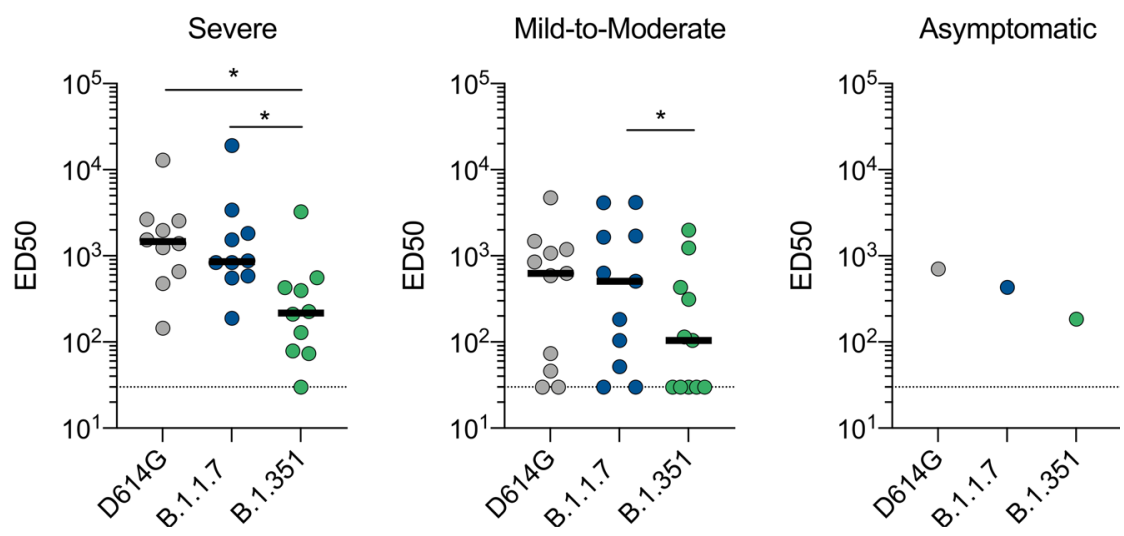

Extended Data Fig. 1 | Sensitivity of SARS-CoV-2 variants to sera from different convalescent individuals. ED50 of neutralization of the D614G, B.1.1.7 and B.1.351. 25 sera from the Orléans Cohort were sequentially sampled at month 3 (M3) and M6 (a and $\mathbf{b}$, respectively) and analyzed as described in Fig. 2 legend. Results are shown as mean from two to four independent experiments. The individuals are classified according to disease severity. The bar indicates the median of values within each column. Two-sided Friedman test with Dunn's multiple comparison was performed between each viral strain at the different time points, ${ }^{*}$ : $p$-value<0.05. M6 (Severe): D614G versus B.1.351 p=0.0417; B.1.1.7 versus B.1.351 p=0.011. M6 (Mild-to-Moderate): B.1.1.7 versus B.1.351 $p=0.0315$. 
Nasal Swabs of vaccinated individuals

a

a VAC\#16

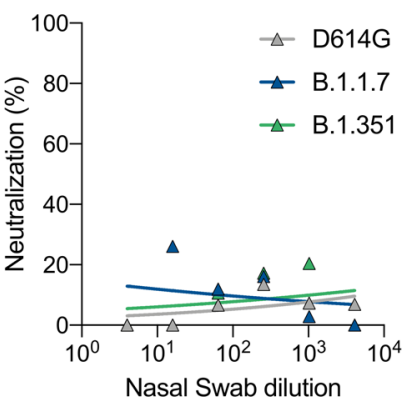

w2 post-vaccination

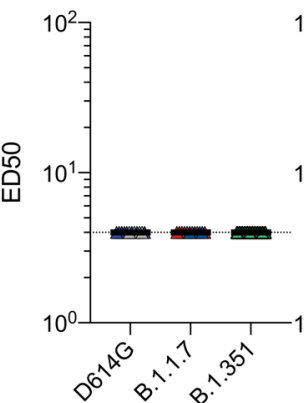
post-vaccination

W4 post-vaccination W6 post-vaccination (W1 post second dose) (W3 post second dose)
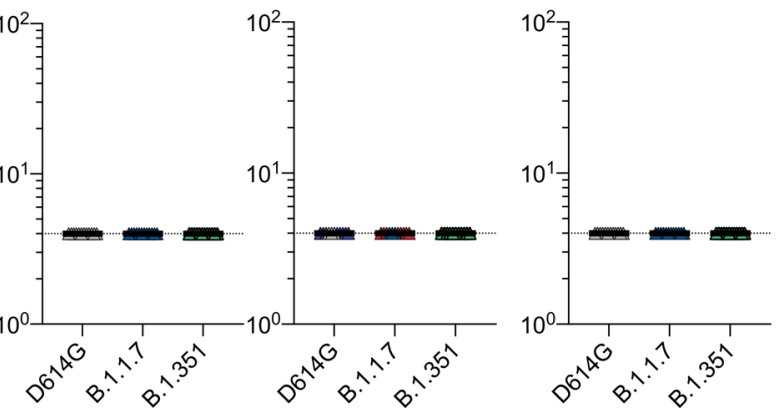

COVID19+ vaccinated individuals
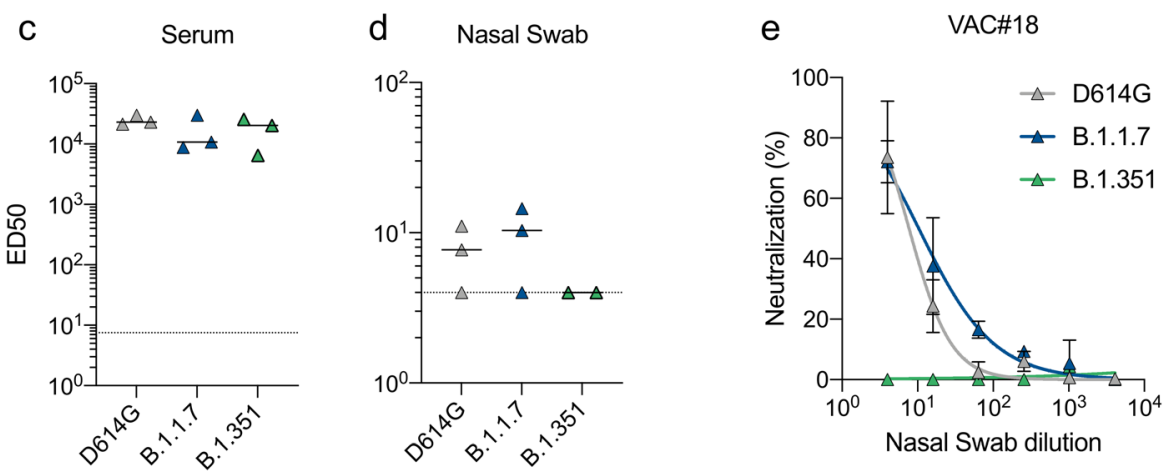

f

W3

post-vaccination

\begin{tabular}{|c|c|c|c|c|c|}
\hline & \multicolumn{2}{|c|}{ Serum } & \multicolumn{2}{|c|}{ Nasal Swab } \\
\hline & & Binding & Neutra & Binding & Neutra \\
\hline ID & 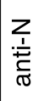 & 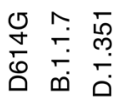 & 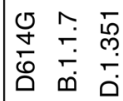 & 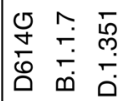 & 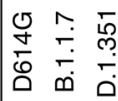 \\
\hline VAC \#1 & - & & & & \\
\hline VAC \#2 & - & & & & \\
\hline VAC \#3 & - & & & & \\
\hline VAC \#4 & - & & & & \\
\hline VAC \#5 & - & & & & \\
\hline VAC \#6 & - & & & & \\
\hline VAC \#7 & - & & & & \\
\hline VAC \#8 & - & & & & \\
\hline VAC \#9 & - & & & & \\
\hline VAC \#10 & - & & & & \\
\hline VAC \#11 & - & & & & \\
\hline VAC \#12 & - & & & & \\
\hline VAC \#13 & - & & & n.d & n.d \\
\hline VAC \#14 & - & & & & \\
\hline VAC \#15 & - & & & & \\
\hline VAC \#16 & - & & & & \\
\hline VAC \#17 & + & & & & \\
\hline VAC \#18 & + & & & & \\
\hline
\end{tabular}

W6 post-vaccination (W2 post second dose)

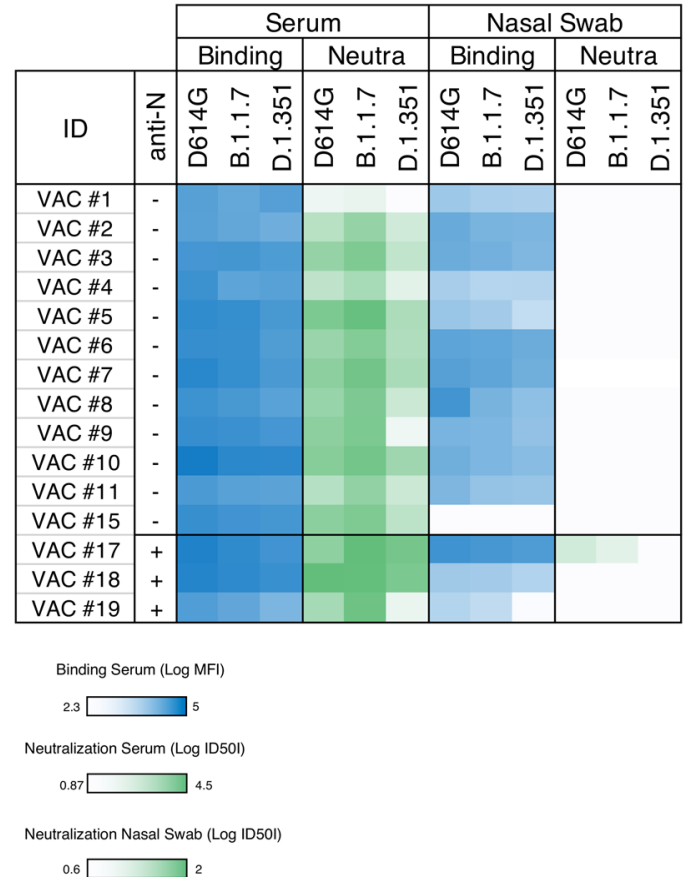

Extended Data Fig. 2 | See next page for caption. 
Extended Data Fig. 2 | Sensitivity of SARS-CoV-2 D614G, B.1.1.7 and B.1.351 variants to nasal swabs from early vaccine recipients. a, Example of neutralization curves with nasal swab from one donor at week 3 (W3) post vaccination. No neutralization was detected. Similar results were obtained at the different times of sampling, up to W6 post-vaccination $\mathbf{b}$, ED50 of neutralization of the three viral isolates, with nasal swabs from 16 vaccine recipients sampled at W2, W3 post first dose and then W1 and W3 post-second dose. The dotted line indicates the limit of detection (ED50 =4). No neutralization was detected. Data are mean from two to four independent experiments. c,d, Analysis of the three COVID-19 vaccinated patients. Neutralizing antibody titers are shown in the serum (dotted line: $E D 50=30$ ) and nasal swab (dotted line: ED50 $=4$ ), at week 2 post vaccination e, Example of neutralization curves with nasal swab from one such COVID-19 vaccine recipient at week 2 post vaccination. Results are shown as mean \pm SD from three independent experiments. f, Heat map summarizing the levels of anti-Spike antibodies and their neutralizing activity in the sera and nasal swabs of the 19 vaccinated individuals. Vac \#17, 18 and 19 were previously infected by SARS-CoV-2. White boxes denote an absence of activity. n.d. = not determined. 


\section{Reporting Summary}

Nature Research wishes to improve the reproducibility of the work that we publish. This form provides structure for consistency and transparency in reporting. For further information on Nature Research policies, see our Editorial Policies and the Editorial Policy Checklist.

\section{Statistics}

For all statistical analyses, confirm that the following items are present in the figure legend, table legend, main text, or Methods section.

$\mathrm{n} / \mathrm{a}$ Confirmed

$\bigotimes$ The exact sample size $(n)$ for each experimental group/condition, given as a discrete number and unit of measurement

$\bigotimes$ A statement on whether measurements were taken from distinct samples or whether the same sample was measured repeatedly

$\triangle$ The statistical test(s) used AND whether they are one- or two-sided

Only common tests should be described solely by name; describe more complex techniques in the Methods section.

$\bigotimes$ A description of all covariates tested

\ A description of any assumptions or corrections, such as tests of normality and adjustment for multiple comparisons

$\triangle$ A full description of the statistical parameters including central tendency (e.g. means) or other basic estimates (e.g. regression coefficient)

AND variation (e.g. standard deviation) or associated estimates of uncertainty (e.g. confidence intervals)

For null hypothesis testing, the test statistic (e.g. $F, t, r$ ) with confidence intervals, effect sizes, degrees of freedom and $P$ value noted Give $P$ values as exact values whenever suitable.

Х $\square$ For Bayesian analysis, information on the choice of priors and Markov chain Monte Carlo settings

$\bigotimes \square$ For hierarchical and complex designs, identification of the appropriate level for tests and full reporting of outcomes

$\bigotimes \square$ Estimates of effect sizes (e.g. Cohen's $d$, Pearson's $r$ ), indicating how they were calculated

Our web collection on statistics for biologists contains articles on many of the points above.

\section{Software and code}

Policy information about availability of computer code

Data collection Harmony Software v4.9 (Perkin-Elmer), Attune Nxt Software v3.2.1 (ThermoFischer), Flowjo Software v10.7.1

Data analysis Excel 365 v16.46 (Microsoft), Prism v9.0.2 (GraphPad Software)

For manuscripts utilizing custom algorithms or software that are central to the research but not yet described in published literature, software must be made available to editors and

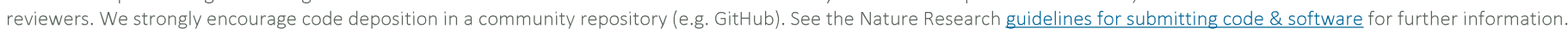

\section{Data}

Policy information about availability of data

All manuscripts must include a data availability statement. This statement should provide the following information, where applicable:

- Accession codes, unique identifiers, or web links for publicly available datasets

- A list of figures that have associated raw data

- A description of any restrictions on data availability

All data supporting the findings of this study are available within the paper and are available from the corresponding author upon request. 
Please select the one below that is the best fit for your research. If you are not sure, read the appropriate sections before making your selection.

\ Life sciences

Behavioural \& social sciences

Ecological, evolutionary \& environmental sciences

For a reference copy of the document with all sections, see nature.com/documents/nr-reporting-summary-flat.pdf

\section{Life sciences study design}

All studies must disclose on these points even when the disclosure is negative.

Sample size $\quad 83$ sera from 58 convalescent individuals, 46 sera and 41 nasal swabs from 19 vaccinees were analyzed in the study. Given the exploratory nature of the study, we did not use statistical methods to predetermine sample size. We included between 15 and 30 patients per group.

Data exclusions

None.

Replication

All experiments were performed and verified in multiple replicates as indicated in their methods/figure legends.

Randomization The experiments were not randomized as we tested all available samples.

Blinding The investigators were not blinded to allocation during experiments and outcome assessment. However, the clinical sampling and biological measurement were performed by different teams. Only the final assembly of the data revealed the global view of the results.

\section{Reporting for specific materials, systems and methods}

We require information from authors about some types of materials, experimental systems and methods used in many studies. Here, indicate whether each material, system or method listed is relevant to your study. If you are not sure if a list item applies to your research, read the appropriate section before selecting a response.

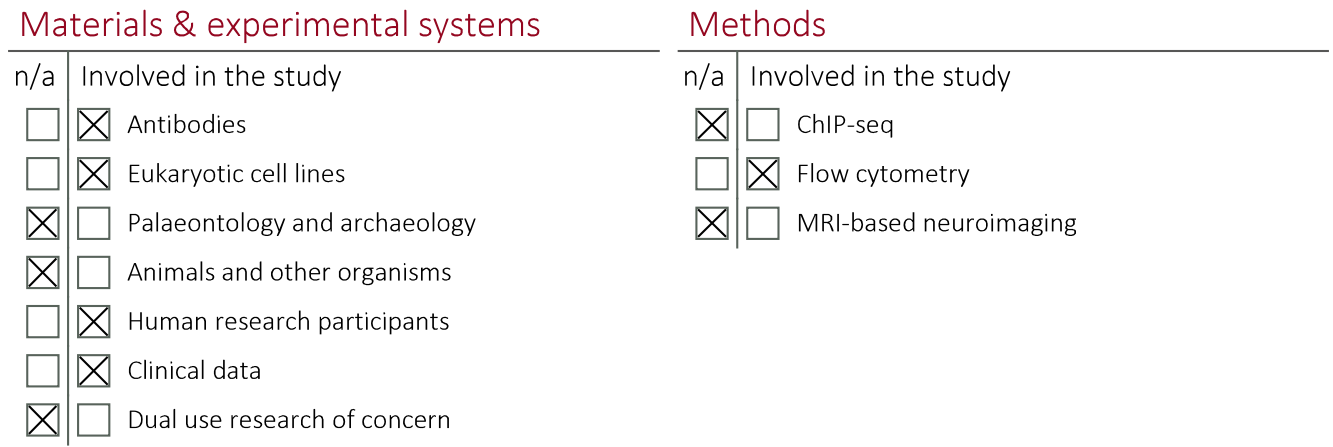

\section{Antibodies}

Antibodies used

mAb10, 48 and 102 are human anti-S monoclonal antibodies isolated and produced by Hugo Mouquet (Institut Pasteur). The Goat anti-Human IgG (H+L) Cross-Adsorbed Secondary Antibody, Alexa Fluor 647 (A21445) was obtained from thermoFisher Scientific.

Validation

mab10,48 and 102 were validated using ELISA binding assays (against the trimeric S, RBD, and S2 proteins) by the team of Hugo

Mouquet. Validation of the goat anti-human IgG is available from the ThermoFisher website.

Eukaryotic cell lines

Policy information about cell lines

Cell line source(s)

Vero E6 (ATCC ${ }^{\circledast}$ CRL-1586 ${ }^{\text {TM }}$ ), 293T cells (ATCC CRL- 3216) and U2OS cells (ATCC ${ }^{\circledR}$ HTB-96 ${ }^{\text {TM }}$ ), were obtained from ATCC. Freestyle 293-F were fromThermoFisher

Authentication

Cell lines were not authenticated

Mycoplasma contamination

All cells are negative for mycoplasma contamination. Tests were performed on a monthly basis

Commonly misidentified lines

(See ICLAC register) 
Policy information about studies involving human research participants

Population characteristics

Orleans' Cohort of convalescent and/or vaccinated individuals: since April 2020, a prospective, monocentric, longitudinal, cohort clinical study enrolling 170 SARS-CoV-2-infected individuals and 30 non-infected healthy controls is on-going, aiming to describe the persistence of specific and neutralizing antibodies over a 24-months period.

Strasbourg Cohort of convalescent individuals: Since April 2020, a prospective, interventional, monocentric, longitudinal, cohort clinical study enrolling 308 RT-PCR-diagnosed SARS-CoV-2 infected hospital staff from the Strasbourg University Hospitals is on-going. Given the exploratory design of the two studies, the characteristics of participants were not preestablished when entering the cohorts. Relevant co-variates are provided in the corresponding supplementary tables.

Recruitment

Orléans cohort : Individuals admitted to the hospital for COVID-19 or with known COVID-19 consulting for a chronic disease were invited to participate.

Strasbourg Cohort : Hospital staff with PCR-confirmed COVID-19 were invited to participate.

Individuals were included without any selection other than those imposed by the entry criteria (known COVID-19 or vaccination). Under these conditions, no particular bias is envisaged.

Ethics oversight

Orléans cohort was approved by national external committee (CPP Ile de France IV, IRB No. 00003835). Strasbourg cohort was approved by the institutional review board of Strasbourg University Hospitals. At enrolment a written informed consent was collected for all participants.

Note that full information on the approval of the study protocol must also be provided in the manuscript.

\section{Clinical data}

Policy information about clinical studies

All manuscripts should comply with the ICMJE guidelines for publication of clinical research and a completed CONSORT checklist must be included with all submissions.

Clinical trial registration

Study protocol

Data collection

Outcomes

\section{NCT04750720 and NCT04441684}

All protocols can be accessed on clinicaltrial.gov

Cohorts started at Hopitaux Universitaires de Strasbourg on April 2020 and Centre hospitalier Régional Orléans on August 2020 and are ongoing.

The primary outcome was the presence of SARS-CoV-2 antibodies binding to the Spike protein (S-Flow assay). The secondary outcome was the presence of neutralizing antibodies (S-Fuse assay)

\section{Flow Cytometry}

Plots

Confirm that:

\The axis labels state the marker and fluorochrome used (e.g. CD4-FITC).

\The axis scales are clearly visible. Include numbers along axes only for bottom left plot of group (a 'group' is an analysis of identical markers).

\All plots are contour plots with outliers or pseudocolor plots.

\A numerical value for number of cells or percentage (with statistics) is provided.

\section{Methodology}

Sample preparation

Freshly prepared $(<24 \mathrm{~h}) 293 \mathrm{~T}$ cells were stained as indicated in the method section. All samples were acquired within $24 \mathrm{~h}$.

Instrument

Attune NxT Acoustic Focusing Cytometer, blue/red/violet/yellow (catalog number : 15360667)

Software

AttuneNxT Software v3.2.1

Cell population abundance

At least 10,000 cells were acquired for each condition.

Gating strategy

All gates were set on 293T cells transfected with an empty plasmid.

$\bigotimes$ Tick this box to confirm that a figure exemplifying the gating strategy is provided in the Supplementary Information. 